\title{
Identification of Road Pavement Types Using Bayesian Analysis and Neural Networks
}

\author{
Joel Preto Paulo \\ ISEL - Instituto Superior de Engenharia de Lisboa, Rua Conselheiro Emdio Navarro 1, 1959-007 Lisbon, Portugal.
}

\begin{abstract}
J. Louis Bento Coelho
Instituto Superior Técnico, Universidade de Lisboa, Avenida Rovisco Pais 1, 1049-001 Lisbon, Portugal.
\end{abstract}

(Received 6 September 2014; accepted 28 February 2017)

A new method to classify and identify different types of road pavements by analysing the near field sound profile and texture using statistical learning methods is proposed. A set of characteristics were extracted from the noise profile and from the road surface texture. Sound measurements were carried out following the close-proximity method with the texture descriptors being provided by a high speed profilometer system. As a first approach, it is assumed that the features extracted from the noise and texture characteristics follow normal distributions. However, this assumption is not completely verified for all types of road surfaces. The method presented herein exploits the use of Bayesian analysis complemented by a neural network in order to improve the classification results.

\section{INTRODUCTION}

Urban road traffic noise is one of the most serious environmental problems that modern societies face nowadays. Efforts have been taken for the development and implementation of noise abatement strategies that can be adopted in urban settings and that do not pose problems of visual impact as noise barriers generally do. Therefore, a considerable variety of different low noise surfaces is now available. ${ }^{1,2}$

A good knowledge of the characteristics of the road pavement surfaces regarding noise and texture is important in terms of assessment for road conformity purposes. Statistical classification and identification of road pavements approaches by using non-destructive methods has been addressed recently. ${ }^{3,4}$ However, these studies focused only on the statistical Bayesian classifier, assuming features such as normal probability density functions and a vehicle speed of $80 \mathrm{~km} / \mathrm{h}$. The possibility of features with different distribution functions and the evolution of each feature set for a range of vehicle speed were left for future study.

The present study aims to outline guidelines regarding an accurate and automatic method for the classification/identification of road pavements using both Bayesian and neural networks classifiers for comparison purposes. $5,6 \mathrm{We}$ believe the use of noise and texture characteristics applied to a pattern recognition approach could be a valuation tool for pavement management systems.

\section{METHODOLOGY}

Macrotexture and sound pressure levels were measured together along the extension of five selected road surfaces in order to extract relevant characteristics to be used on the pavement classification.
The pavement surface macrotexture was estimated by the mean profile depth (MPD) method. The pavement surface data was acquired with a high speed Profilometer (HSP) following the ISO 13473-1 standard. ${ }^{7}$ The sound signal was acquired, in near field conditions, along the road segments for vehicle speeds in the range of 30 to $100 \mathrm{~km} / \mathrm{h}$ (in most tracks), in steps of $10 \mathrm{~km} / \mathrm{h}$, following the close proximity procedure (CPX) as described in the ISO/DIS 11819-2 standard. ${ }^{8}$

For final model validation purposes, only the speed of $80 \mathrm{~km} / \mathrm{h}$ was used, which was chosen among the reference speeds mentioned in the ISO/DIS 11819-2 standard. The tyre used was Michel-in Energy 205/65-R15, designated as standard reference test tyre (SRTT). It is one of the two tyres recommended in the proposed ISO/DIS 11819-2 standard.

From the macrotexture and the corresponding audio signal of each road surface, a set of features that best described the type of data acquired were selected and analysed. The relation macrotexture/noise was determined through this set of features which in turn was used for evaluation and assessment of each road surface.

After applying the feature selection procedure to the whole set of the features considered relevant on characterizing the road surface, a sub-set was applied to the Bayes classifier and a neural network. Figure 1 shows the scheme of the setup used in this research.

The road surface set chosen for this study included the ones most commonly used in Portugal: one of dense asphalt (DA), one of slurry surfacing (SS) and three surfaces of open-graded asphalt rubber (OGAR), which are used extensively now. ${ }^{9}$ Table 1 presents the maximum aggregate size and the age of each surface. The maximum aggregate size used together with the texture and the porosity as well as the age of the surface are reported in literature as being the main parameters affecting tyre/surface noise. ${ }^{10-14}$ Although this set does not cover the 


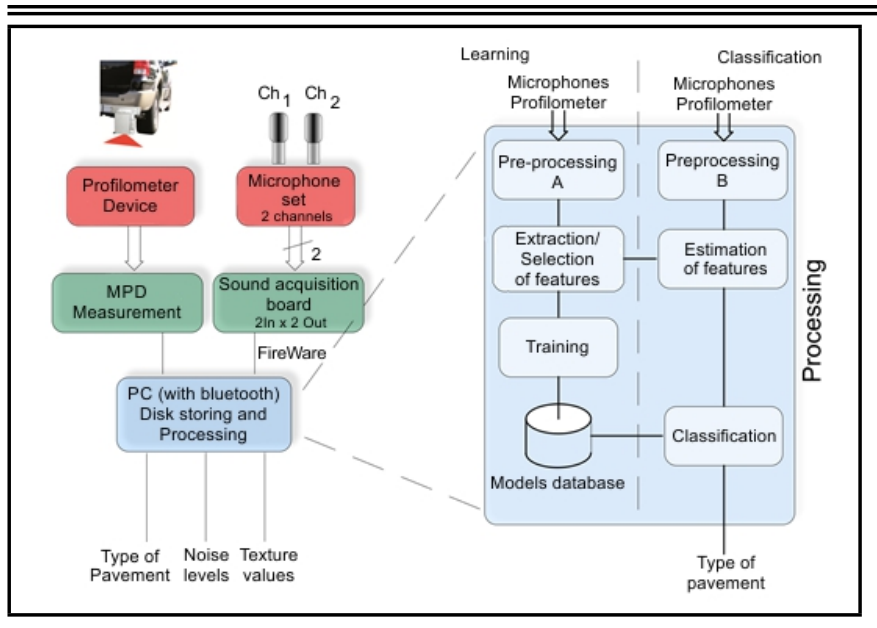

Figure 1. Scheme of the experimental and analysis setup showing details of the classifier.

Table 1. Properties of the asphalt mixes

\begin{tabular}{|l|c|c|c|c|c|}
\hline Type of mix & $\begin{array}{c}\text { OGAR1 } \\
\text { (type 1) }\end{array}$ & $\begin{array}{c}\text { SS } \\
\text { (type 2) }\end{array}$ & $\begin{array}{c}\text { OGAR2 } \\
\text { (type 3) }\end{array}$ & $\begin{array}{c}\text { OGAR3 } \\
\text { (type 4) }\end{array}$ & $\begin{array}{c}\text { DA (0/16) } \\
\text { (type 5) }\end{array}$ \\
\hline $\begin{array}{l}\text { Maximum aggregate } \\
\text { size (mm) }\end{array}$ & 10 & 12 & 10 & 10 & 16 \\
\hline Age (years) & 1 & 4 & 4 & 2 & 1 \\
\hline
\end{tabular}

majority of road surface types and age conditions, they are enough to prove the concept of identification of road pavement types using different classifiers.

\section{PAVEMENT FEATURES}

The features characterizing the road pavement were extracted from measurements of texture based on the MPD and of sound levels. The sound pressure level is the feature that best describes the type of surface, given its close relationship with the noise energy level perceived by the human auditory system. However, the sound pressure is highly dependent on several factors such as the speed of the vehicle, the type of tyres and the age of the pavement. Therefore, a combination of features is necessary.

The following sections describe the vector of features adopted in this work. It includes a set of characteristics which are the most appropriate to relate noise and texture, after applying the feature selection procedure. This procedure has several goals: (i) to remove any redundant characteristics, (ii) to decrease the dimensionality of the feature vector and (iii) to decrease the computational load of the classifier. The selection method consisted of a PCA approach. Nevertheless, the selected vector of features has to include all the fundamental characteristics of the road surface relevant to the classification task.

Figure 2 depicts the ranking for the features (by backward searching method and based on the inter-intra criterion) originally introduced in the texture and noise correlation study. ${ }^{15-17}$ The procedure of ranking the features is important to select the most valuable features in order to derease the computational load of the classification/identification process. These features are shown in Table 2.

The features are sorted in descending order of relevance as

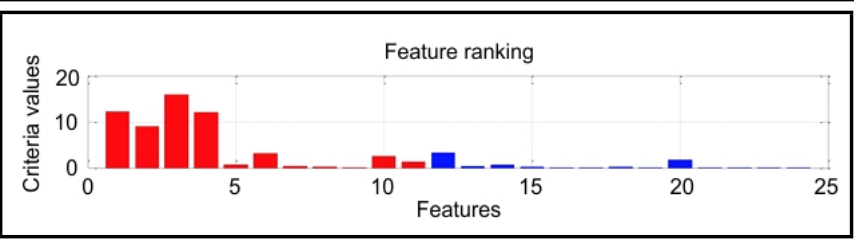

Figure 2. Rank of the noise (red bars) and texture (blue bars) features for classification proposals.

Table 2. Pavement selected features

\begin{tabular}{|l|l|}
\hline Feature name & Obs. \\
\hline LAeqFull & overall sound pressure levels \\
\hline LAeqLo & low frequency band \\
\hline LAeqMid & middle frequency band \\
\hline LAeqHi & high frequency band \\
\hline Ct & centroid \\
\hline CtLo & centroid low frequency band \\
\hline CtMid & centroid middle frequency band \\
\hline CtHi & centroid high frequency band \\
\hline FreqMaxSPL & frequency band of maximum SPL \\
\hline maxLAeqBand & maximum SPL band \\
\hline max $2 F r e q B a n d$ & secondary maximum SPL band \\
\hline meanMPD & mean of MPD \\
\hline stdMPD & standard deviation of MPD \\
\hline CtMPD & centroid MPD \\
\hline CtLoMPD & centroid MPD low frequency band \\
\hline CtMidMPD & centroid MPD middle frequency band \\
\hline CtHiMPD & centroid MPD middle frequency band \\
\hline maxAmpMPD4 & maximum amplitude above mean of MPD \\
\hline rmsmaxAmpMPD & maxima rms of MPD \\
\hline maxMPD & maxima of MPD \\
\hline CrestF_ MPD & Crest Factor of MPD \\
\hline pk2pkMPD & peak-to-peak of MPD \\
\hline ZCrossMPD & zero crossings of MPD \\
\hline meanCorrMPD & mean correlation MPD \\
\hline
\end{tabular}

$\{314212610201114513715818242291923$ $1721 \quad 16\}$. As observed, the features extracted from noise have more significance, are more decorrelated from the others than the features from texture. Effectively, the MPD method does not address some surface texture characteristics such as the pavement aggregate particle shape, size and distribution. ${ }^{18}$ The method is not meant to provide a complete assessment of pavement surface texture characteristics.

Despite these considerations, several characteristics defining different pavements can be selected.

\subsection{Noise Characteristics}

The A-weighted RMS sound pressure is the acoustic feature that, at a first approach, best describes the type of surface. ${ }^{?, 19,21}$ For this reason, the A-weighting curve was applied to the acquired sound signals. This procedure has a further advantage of removing the low frequency band energy, generated mostly by the moving vehicle's air flow. The A-weighted sound pressure level should thus be the primary noise feature to be used. There are scores of generation mechanisms that in turn lead to a plethora of tyre/road noise scenarios. Therefore, deciding which modelling strategy to use is rather difficult. The issue is even more problematic because of the 
complexity of the radiation conditions for both vibration and aerodynamic sources. Therefore, the noise spectrum must be split into several complementary frequency bands to monitor the tyre/pavement noise generation phenomena so that the performance of the classifier is enhanced. In this study, three frequency bands were used: a low band $(200-1400 \mathrm{~Hz})$, a middle band $(1400-3000 \mathrm{~Hz})$ and a high band $(3000-8000 \mathrm{~Hz})$. Additionally, a $1 / 3$ octave band analysis was considered in some cases, for improved frequency discrimination. Full band analysis was also used to account for overall noise energy. In order to improve the results in the classification stage and for comparison purposes, a smoothing technique was applied.

The noise features selected from a subset of the first 12 features of the rank, given by Fig. 2, are as follows:

(1) Overall sound pressure level - LAeqFull (in dBA), in the range of 200 to $8000 \mathrm{~Hz}$. Most sound energy remains in this frequency band. This feature is estimated by the moving average method.

(2) Narrow band sound pressure levels: low band LAeqLo (200-1400 Hz), middle band - LAeqMid $(1400-3000 \mathrm{~Hz})$ and high band - LAeqHi (3000$8000 \mathrm{~Hz}$ ). This feature splits the audio frequency band of interest in three parts for better discrimination.

(3) Spectral energy centroid - Ct. The spectral centroid is commonly associated with the measure of the "brightness' of a sound. This measure is obtained by evaluating the 'centre of gravity' using the Fourier transform's frequency and magnitude information. The individual centroid of a spectral frame is defined as the average frequency weighted by amplitudes, divided by the sum of the amplitudes. The computation of this feature is based on the squared magnitude of the spectrum using the shorttime Fourier transform (STFT) and performed frame-byframe along the time axis. The use of the squared magnitude of the spectrum, a measure of the noise energy, is aimed at getting an improved correlation with the stimulus perceived by the human auditory system.

(4) Spectral band energy centroid - CtLo, CtMid and CtHi. It is same as $\mathrm{Ct}$ computed for three frequency bands.

(5) Third octave band sound pressure levels - Oct13B.

(6) Spectral Energy Maximum - FreqMaxLAeq. This feature corresponds to the main peak of the power spectral density estimate. The tyre/road pavement noise spectrum profile is characterized often by a maximum sound intensity centred in the range of about 700 to $1200 \mathrm{~Hz}$. This feature is evaluated by averaging $\mathrm{N}$ consecutive frames (moving average method) in the frequency domain.

\subsection{Texture Characteristics}

The criteria adopted for choosing the set of texture features was based on the physical meaning of the data given

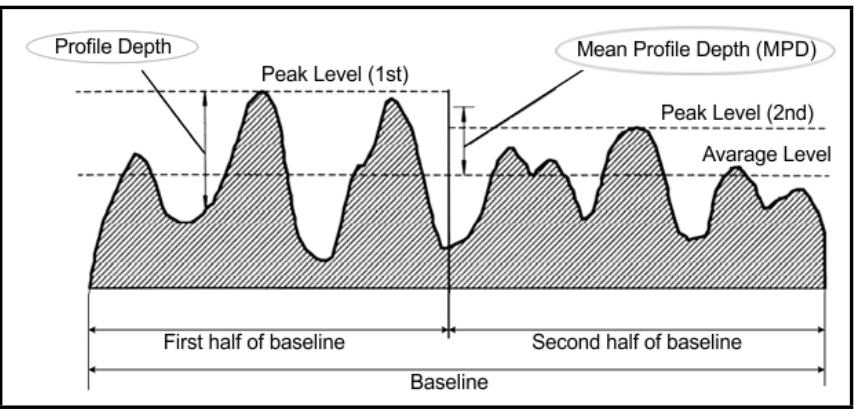

Figure 3. Scheme for the calculation of MPD.

by the MPD. The MPD is used to characterize the macrotexture of pavements, giving a measure of the mean of the profile depth only. The following development gives a step forward concerning this issue. The estimation of MPD is given by $M P D=\left[\right.$ peaklevel $\left.\left(1^{\text {st }}\right)+\operatorname{peaklevel}\left(2^{\text {nd }}\right)\right] / 2-$ Average level, after the ISO 13473-1 standard. The diagram depicted in Fig. 3 illustrates the concept.

In order to evaluate the characteristics of the MPD in a number of simulations of different textures, a simple model was developed to generate the longitudinal pavement profile. The model is able to generate two types of profiles: the first type is assigned to periodic profiles based on the selected wavelength and the second type consists of generating the profile randomly using a table with the pavement grading curves that gives information about the grade percentage of the aggregates. Some examples of the type of aggregates considered are round shaped, triangular shaped, raised cosine shaped and square shaped.

Since all parameters extracted are mean values, the best texture features (selected from a sub-set of the first 12 features of the rank, given by Fig. 2) to be analysed in this study are as follows:

(7) Mean of MPD, meanMPD (in mm) - it represents the mean value of the MPD using the moving average method.

(8) Standard Deviation of MPD, stdMPD (in mm) - it provides information about the regularity of the surface.

(9) Centroid MPD, CtMPD - this feature gives information about the centre of gravity of the spectral texture.

(10) Maxima of MPD, maxMPD (in mm) - it corresponds to the absolute maxima of the MPD.

\subsection{Evolution of Features with Vehicle Speed}

The complete analysis of the features should comprise the dependence of each feature with the speed of the moving vehicle. Therefore, the characterization/identification of a pavement can be evaluated for one specific speed only. Although for a complete analysis three velocities should be used, as recommended by the ISO/DIS 11819-2, in some circumstances, such procedure is difficult to implement due to certain factors like the logistics associated to in situ sound measurements. Figures 4-5 show a set of features and their dependence on 


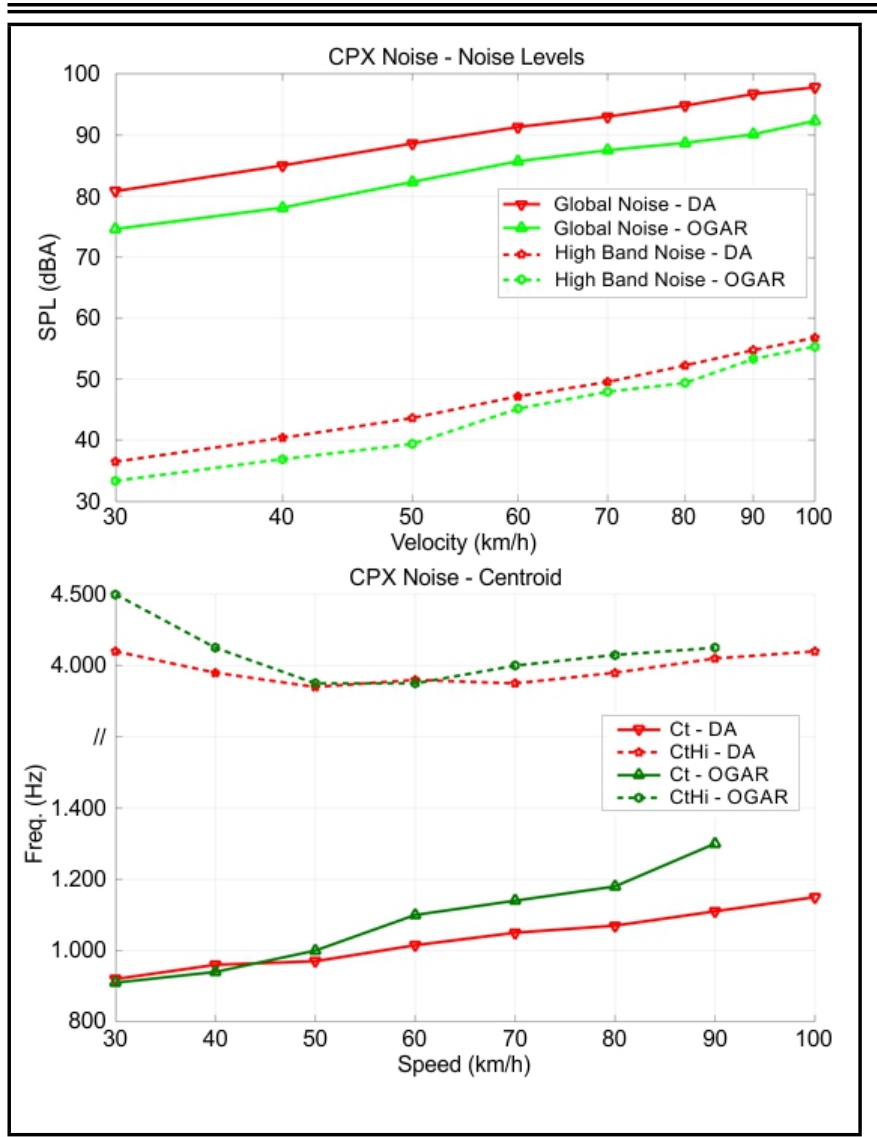

Figure 4. Long-term and high frequency band noise levels and centroid for different vehicle speeds.

the vehicle speed. As a matter of simplicity, only the DA and OGAR1 pavements are shown for comparison purposes, since they have the same age.

Previously published works have mentioned that the sound pressure levels follow a linear evolution with the logarithm of the speed. ${ }^{2,3}$ Although this is true for the long-term noise levels, a deviation is observed for the high frequency bands, as shown in Fig. 4, at least for these types of pavements. This observation is confirmed for the centroid feature. In fact, it should be a horizontal line.

As shown in Fig. 5, although the long-term sound pressure levels, which are strongly dependent on the frequency band for higher levels, follow a linear fashion with the decimal logarithm of the speed, this is not true for the high frequency bands, which reveals a linear dependence on speed. As observed, an increase of the speed implies an increase on $C t$ feature, following a non-linear law. In fact, the offset on the spectra is frequency band dependent.

\section{CLASSIFIER PROCESSING}

The classifier system is trained using the MPD and sound features for each type of pavement. Therefore, the module used for classification was the result of a supervised learning approach. A database populated by entries describing different types of surfaces was built beforehand. ${ }^{1}$ The road pavement classification framework consists mainly of two stages: (i) feature extraction and (ii) classification.

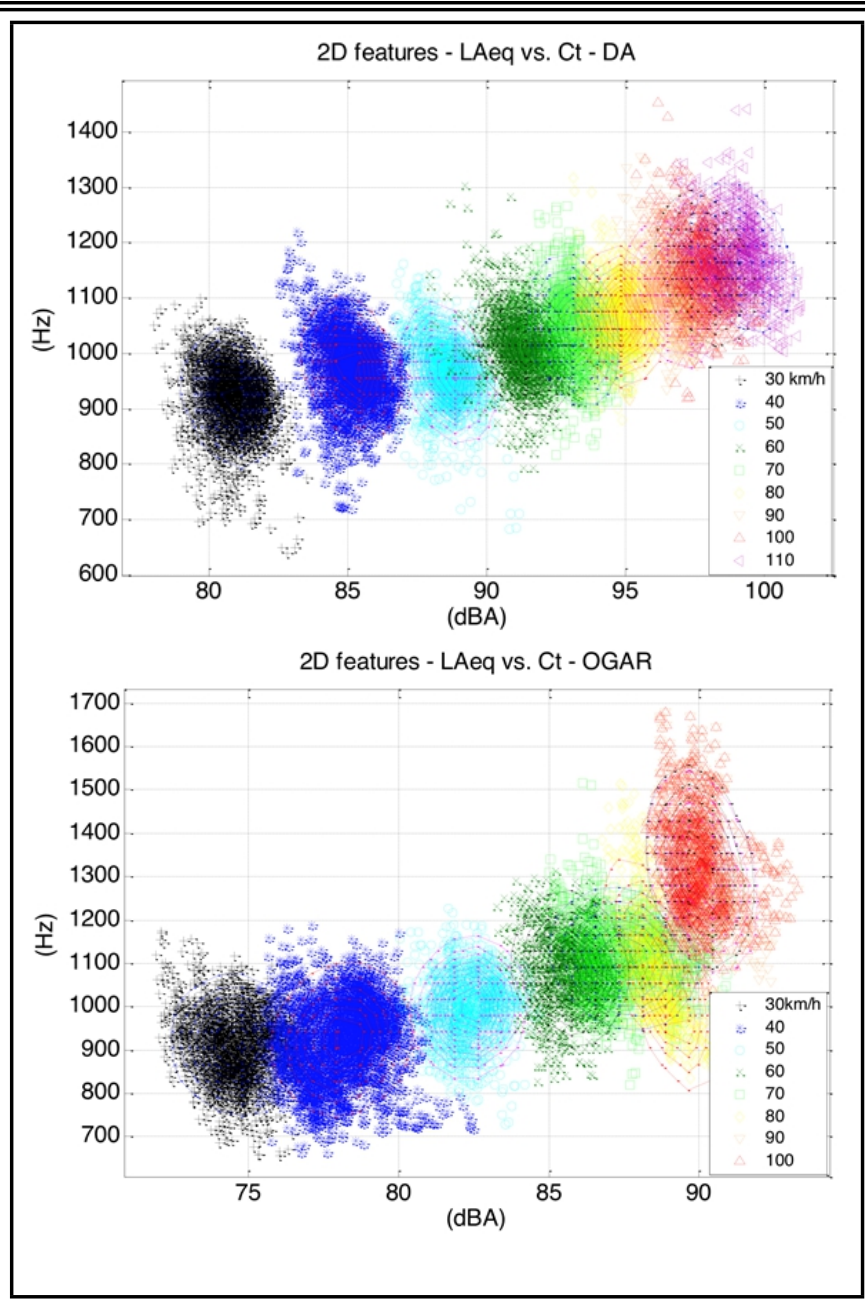

Figure 5. 2D feature space of LAeq vs. Ct for different vehicle speeds and for the pavements dense asphalt (DA), left and open-graded asphalt rubber (OGAR1), right. The contours give a measure of the density of the observations on the feature space.

The data samples are classified in a specific class depending on its location on the feature space. ${ }^{6}$ In this study, 12 features were considered for testing.

\subsection{Features Distribution}

Figure 6 shows the density functions associated to LAeq and $\mathrm{Ct}$ features providing guidelines to choose the most convenient type of classifier. Similar results can be found for the remaining features.

As suspected from the observation of the contours in Fig. 5 and confirmed by Fig. 6, the density functions of the features for OGAR1 pavement do not follow a normal distribution for some types of pavements. Therefore, Bayesian and neural network classifiers were tested for comparison and complementation. Moreover, excluding the results for speeds up to $50 \mathrm{~km} / \mathrm{h}$, due to the influence of the engine noise of the vehicle, the density functions do not exhibit the same value for $C t$ feature and different shapes for LAeq are shown.

\subsection{Classifier Learning}

The feature vectors for each pavement class were divided into two datasets: a training set and a test set. A randomly 


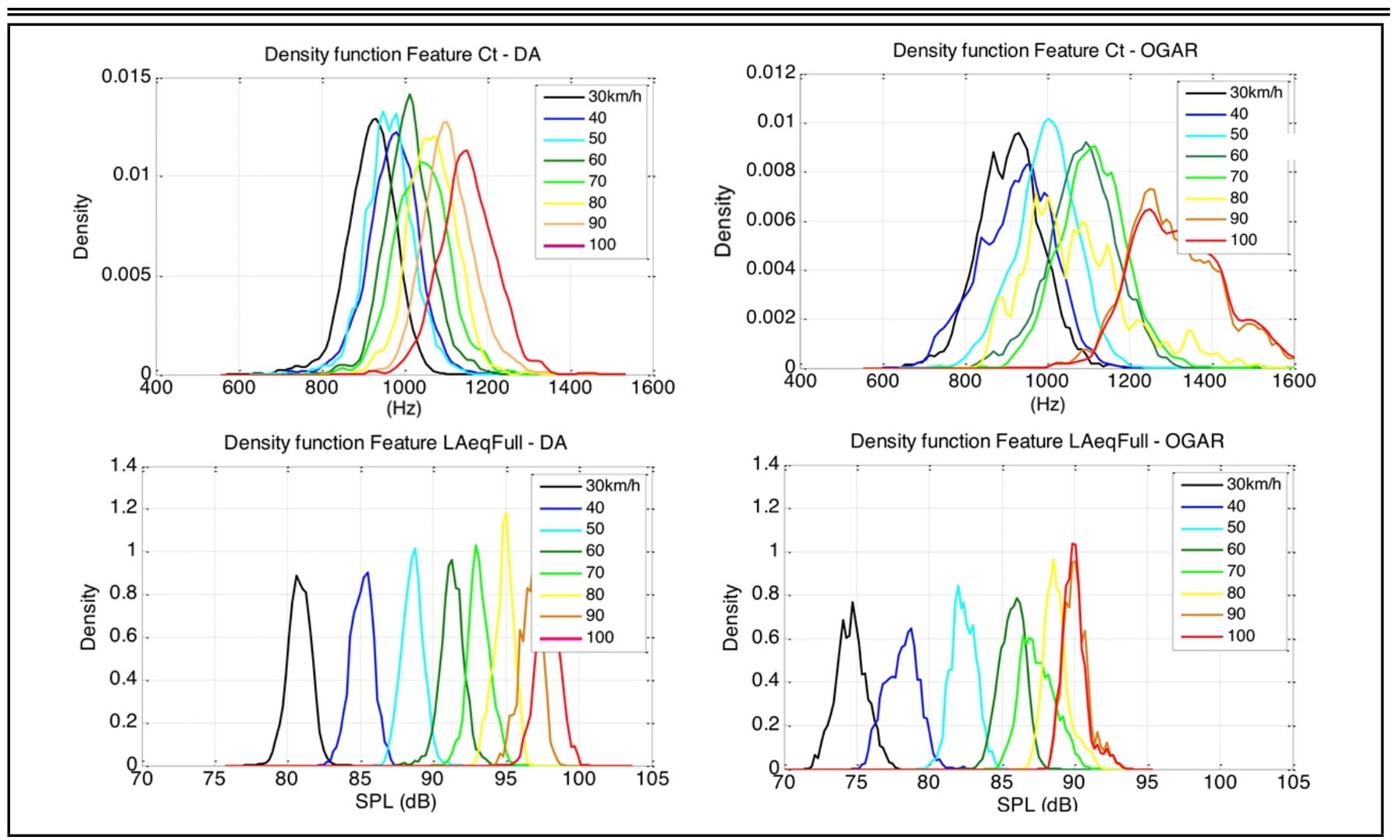

Figure 6. Density functions of feature LAeq and Ct for different vehicle speeds.

chosen subset of $30 \%$ of the vectors is assigned to the training set and the remaining $70 \%$ correspond to the test set. $^{21}$ The Bayesian classifier corresponds to a quadratic discriminant analysis (QDA) and the neural network classifier is a feedforward network with tansigmoid transfer functions for both the hidden layer, which uses 9 neurons and output layer. An $N$-dimensional density model (where $N$ is the length of the feature vector) is then adjusted to each class, based on the training set. $^{22}$

One advantage of using the neural networks against Bayesian classifiers is that the assumption of normal distribution for the selected features is not required.

\section{EXPERIMENTAL RESULTS}

The features used in this experiment were obtained for frames of equal 2048 samples length using rectangular or Hanning sliding windows, depending on the analysis. ${ }^{23,24}$ The sliding factor was $12.5 \%$ of the window length (hop-size of 256 samples), with sampling frequency of $32 \mathrm{kHz}$. The use of this sliding factor (lower than 50\%) allows a better identification of the variability of the pavement texture and location of any surface irregularities. In order to make the sampling rate for texture and for noise measurements the same, a resampling technique was applied to the texture data (the data provided by the MPD measurements is 40,000 samples $/ \mathrm{km}$, one sample for each $2.5 \mathrm{~cm})$ for a vehicle speed of $80 \mathrm{~km} / \mathrm{h}(\mathrm{R}=36)$.

The result of the pavement classification is assessed by means of the related confusion matrix shown in Fig. 7, for OGAR1 (type 1), SS (type 2), OGAR2 (type 3), OGAR3 (type 4) and DA (0/16) (type 5) road surfaces. This matrix is obtained with the test dataset and yields the error committed by the classifier. This is a square matrix, with dimension equal to the number of classes, in which each entry, say $\left(k_{1}, k_{2}\right)$, is an estimate of the probability that an observation (from true) pavement class $k_{1}$ is classified as belonging to class $k_{2}$. The principal diagonal of the confusion matrix corresponds to the correct pavement classification. Thus, the confusion matrix for the ideal classifier consists of a diagonal matrix, with only the elements on the main diagonal of the confusion matrix being non-zero. The numeric values in each cell give the result of classification in percentage of samples with true pavement classification.

In order to compare the performance of the Bayes classifier against the neural network classifier, the analysis was performed using two different sets of features: (i) a number of selected features and (ii) the complete set of features. In Fig. 7, the first value in each cell is for the selected set of features and the second shows the result for all features considered in Table 2. The results showed that for both classifiers, the pavements OGAR1 and SS are well identified.

Bayes classifier: the pavement OGAR2 is barely identified with the set of 12 selected features, about $79 \%$ classified on the true pavement, increasing to $91 \%$ with all features. With all features (except the OGAR2 type) the true pavement is identified with a degree of confidence above $95 \%$.

Neural network: a degree of confidence above $90 \%$ is observed for the 12 selected features, for all pavements and above $96 \%$ using all the features. The OGAR2 type (pavement 3 ) is well identified with this classifier.

As a conclusion, the neural network classifier leads to bet- 


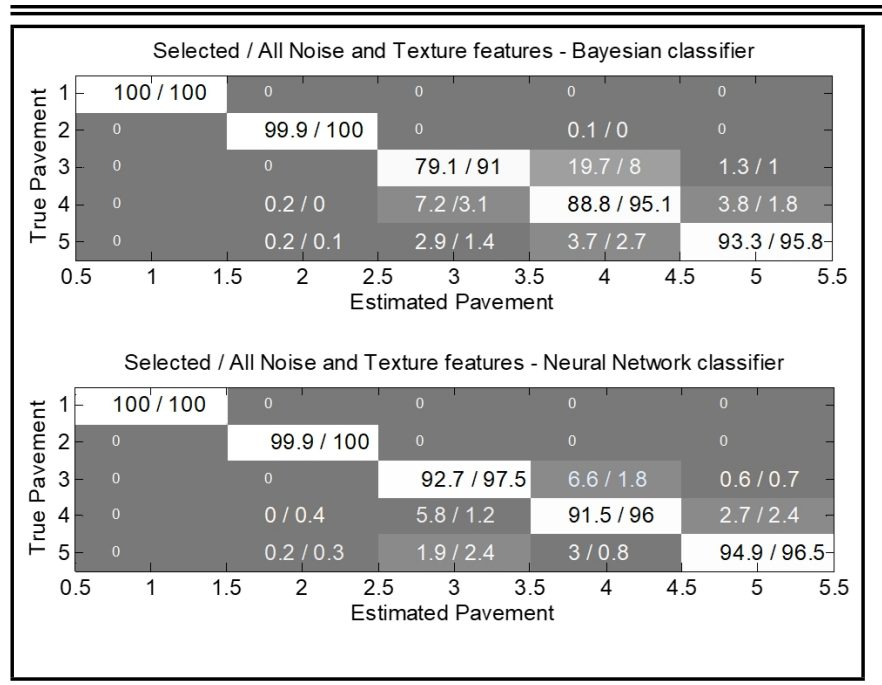

Figure 7. Confusion matrices using the Bayesian (top) and neural network (bottom) classifiers. Each non-zero cell holds the result for the selected features (first 12 features given by the ranking method) and for all the set of features (24 features).

ter results when compared with the Bayesian classifier. Although the increase of the amount of different road pavement types could alter the classification results slightly, this conclusion will remain true since the neural network does not need normal distributions for the features used.

Advantages of the Bayesian classifier over the neural networks are the time consumed for the training process, about 1:100 (all features) and 1:50 (12 selected features) and its stability. However, after the training stage, the neural network classifier is very fast on the stage of classification. Moreover, a normal distribution for some types of pavements is not verified, as shown in Fig. 6, degrading the results.

\section{CONCLUSIONS}

A method for classification and identification of different types of road pavements was developed and implemented. The method was designed using statistical classification techniques comprising an array of procedures to acquire and pre-process road sound signals near a test tyre and macrotexture information provided by a profilometer device.

The Bayesian and the neural network classifiers were compared for the purposes of classification and identification of different types of road pavements. The method uses features extracted from surface texture and the noise resulting from the traffic of light motor vehicles.

Tests were conducted with a set of five types of pavements, including currently used dense, porous and gap-graded asphalts, for different vehicle speeds.

The results of road surface classification showed in general a very good agreement with the true road pavements for the two classifiers assessed, above $90 \%$ for the Bayesian and above $95 \%$ for the neural network. This remarkable result proves the concept of using statistical classification approaches applied to road pavements. Moreover, with a feature selection procedure, the resulting accuracy of the neural network classifier is significantly better than with the Bayesian classifier, achieving about
$100 \%$ of true identification pavement for all pavements tested. This is due to the fact that the assumption of features with normal distribution is incorrect for some types of road pavements. However, the training step time is much longer and the preprocessing step of the data used to define the features in order to obtain the correct neural network parameters should be a lot more careful. Moreover, with the use of smoothing techniques applied to the features an improvement in the results is expected.

In order to evaluate the dependence of the vehicle speed on the feature set, curve fitting methods were applied to some features. It was found that the increase of sound pressure levels for high frequency bands do not follow a linear behaviour with the decimal logarithm of the speed, which occurs for the long-term noise levels. The same result is observed for the $C t$ feature. This type of conclusion is useful in the estimation of features for a specific speed other than the reference speed.

The method can be used for assessing road pavements in terms of age and degree of erosion. The procedure offers a good and accurate characterization of the road surface noise emission and provides valuable data both for low noise pavement designers and for road engineers, builders and contractors. This method can also be used for conformity tests on new road pavements, by comparing it with accurate data from a reference road surface. A further application of this road pavement classification system is the improvement of accuracy of road pavement data for road traffic noise modelling and mapping purposes.

Due to the discrepancy of measurement data for some types of pavements, care must be taken with the pre-processing of the data, excluding, as much as possible, discrepancies related to the problems of construction/degrading process of the roads (unless the goal of the study is con-formity road assessment).

Since the speed of the vehicle is related to the resulting noise levels, the correct surface classification should be carried out with measurements at several speeds and at constant velocity.

In order to evaluate the dependence of the vehicle speed on the feature set, curve fitting methods were applied to some 2D feature space.

Finally, one can conclude that features based on the MPD are insufficient to describe the texture accurately. As a final remark, the correct correlation between noise and texture characteristics should take into account not only the level of macrotexture but also the microtexture and megatexture in order to provide more information to correlate texture and noise conveniently, thus achieving better classification results.

\section{ACKNOWLEDGMENTS}

Research was partially supported by the FCT-CAPS/IST pluriannual funding through the POS_C Program that includes FEDER funds and by the FCT CAPS-IST-ID strategic project and by PROTEC ISEL/IPL funds from the Ministry of Science, Technology and Higher Education of Portugal. 


\section{REFERENCES}

1 Morgan, P. (Ed.). Guidance manual for the implementation of low-noise road surfaces, benefits, 769, 66, (2006).

2 Kuijpers, A., \& Van Blokland, G. Simulation tool for road/tyre modelling — The influence of road parameters on tyre/road noise, SILENCE Project, (2006).

3 Descornet, G. and Goubert, L. Noise classification of road pavements, Task 1: technical background information 1, Draft Report 05, Directorate-General Environment, European Commission, (2006).

4 Anfosso-Lédée, F. Modeling the local propagation effects of tire-road noise: propagation filter between CPX and CPB measurements, Proc. Internoise, Prague, Czech Republic (2004).

5 Preto Paulo, J., Freitas, E., Bento Coelho, J. L. Texture and noise features for road pavement identification and classification, Proc. InterNoise10, Lisbon, Portugal, (2010).

6 Paje, S. E., Bueno, M., Viñuela, U., and Terán, F. Toward the acoustical characterization of asphalt pavements: Analysis of the tire/road sound from a porous surface, J. Acoust. Soc. Am. 125, 5-7, (2009). https://dx.doi.org/10.1121/1.3025911

7 Norma, I. S. O. 13473-1-Characterization of pavement texture by use of surface profiles. Part 1: Determination of Mean Profile Depth, 1, (1997). https://dx.doi.org/10.3403/03027853u

8 BS EN ISO 13473-1: Characterization of pavement texture by use of surface profiles. Part 1: Determination of Mean Profile Depth-1, (2004). https://dx.doi.org/10.3403/03027853u

9 Freitas, E., Preto Paulo, J. and Bento Coelho, J. L. Silent surfaces: An experience in portugal, Proc. SURF, Portoroz, Slovenia, In CD-ROM, (2008).

10 Kropp, W., Kihlman, T., Forssén, J. and Ivarsson, L. Reduction potential of road traffic noise, Royal Swedish Academy of Engineering Sciences, report, (2007).

11 Licitra, G., Tetic, L. and Cerchiai, M. A modified Close Proximity method to evaluate the time trends of road pavements acoustical performances, Applied Acoustics, 76, 169-179, (2014). https://dx.doi.org/10.1016/j.apacoust.2013.07.017

12 Bendtsen H., Lu Q., and Kohler E. Technical Report UCPRC-RP-2010-01, Acoustic aging of asphalt pavement: A Californian Danish Comparison, (2009)

13 Ibarra, D., Cobo, P., Calvo, J.A. and San Román, J.L. Relating the near field noise of passenger cars with the driving behavior, Noise Control Engineering Journal, 60 (2), 171183, (2012). https://dx.doi.org/10.3397/1.3682961
14 Licitra, G., Cerchiai, M., Teti L., Ascari, E., Fredianelli, L. Durability and variability of the acoustical performance of rubberized road surfaces. Applied Acoustics 94, 20-28, (2015). https://dx.doi.org/10.1016/j.apacoust.2015.02.001

15 Wilsona, S.P., Harrisb, N.K. and OBrien, E.J. The use of Bayesian statistics to predict patterns of spatial repeatability, Transportation Research Part C: Emerging Technologies, 14 (5), 303-315, (2006). https://dx.doi.org/10.1016/j.trc.2006.07.002

16 Cana, A., Dekonincka, L., Rademakerb, M., Van Renterghema, T., De Baetsb, B. and Botteldooren, D. Noise measurements as proxies for traffic parameters in monitoring networks, Elsevier, Science of The Total Environment, 410-411, 198-204, (2011). https://dx.doi.org/10.1016/j.scitotenv.2011.09.053

17 Sandberg, U. and Ejsmont, J. A. Tyre/Road noise reference book, Informex, Kisa, Sweden, (2002).

18 Sayers M. and Karamihas, S. The little book of profiling, University of Michigan, (1998).

19 Preto Paulo, J., Bento Coelho, J. L., and Figueiredo, M. A. T. Statistical classification of road pavements using near field vehicle rolling noise measurements, Journal of the Acoustical Society of America, 128 (4), 1747-1754, (2010). https://dx.doi.org/10.1121/1.3466870

${ }^{20}$ Lui W. K., and Li, K. M. A theoretical model of the horn amplification of sound radiated from tires above a porous road pavement, J. Acoust. Soc. Am. 116, 313-320, (2004).

21 Li, D., Sethi, I. K., Dimitrova N. and McGee T. Classification of general audio data for content-based retrieval, Pattern Recognition Letters, 22, 533-544, (2001).

22 Herişanu, N., and Bacria, V. Some effects of rubberized asphalt on decreasing the phonic pollution, In Applied Mechanics and Materials, Trans Tech Publications, 430, 257-261, (2013). https://dx.doi.org/10.4028/www.scientific.net/amm.430.257

23 Paje, S. E., Bueno, M., Viñuela, U., and Terán, F. Toward the acoustical characterization of asphalt pavements: Analysis of the tire/road sound from a porous surface, J. Acoust. Soc. Am., 125 (1), 5-7, (2009). https://dx.doi.org/10.1121/1.3025911

24 Hastie, T., Tibshirani, R., and Friedman, J. The elements of statistical learning, Springer-Verlag, New York, (2001). https://dx.doi.org/10.1007/b94608 